\title{
Descargas eléctricas y sus aplicaciones
}

\section{Electrical discharges and applications}

\author{
Héctor Jaime Dulce-Moreno ${ }^{1}$
}

Forma de citar: H. J. Dulce, Descargas eléctricas y sus aplicaciones, Eco.Mat. 2015; 6(1): 6-21.

Recibido:

Abril 22 de 2015

\section{Aceptado:}

Junio 26 de 2015

\section{RESUMEN}

Las descargas eléctricas han sido, para el hombre, inicialmente motivo de admiración, posteriormente objeto de estudio, y finalmente de aplicación. Este ciclo se ha repetido a lo largo de la historia de la humanidad. Las primeras descargas eléctricas conocidas por el hombre fueron las naturales, denominadas descargas atmosféricas o más comúnmente rayos; uno de los efectos más importantes de las descargas atmosféricas fue la formación de incendios; incluso, para algunos autores, fue la forma como nuestros antepasados conocieron el fuego. Existen múltiples ejemplos desde los filósofos griegos, pasando por los científicos del siglo dieciocho, que se inspiraron en las descargas atmosféricas para construir sus teorías, por ejemplo las teorías sobre la carga eléctrica, que terminaron en aplicaciones de tipo tecnológico.

Palabras clave: descargas eléctricas

\begin{abstract}
Electrical discharges have been, for man, initially cause for admiration, then under consideration, and finally application. This cycle has been repeated throughout the history of humanity. The first electrical discharges known to man are called natural discharges, known more commonly atmospheric discharge or lightning; one of the most important effects of lightning was the formation of fire; even, for some authors, it was the way our ancestors knew fire. There are many examples from the Greek philosophers, scientists through the eighteenth century, who were inspired by the atmospheric discharge to build their theories, such as theories of electric charge, which ended in such technological applications.
\end{abstract}

Keywords: electrical discharge
${ }^{1}$ Doctor en Ciencias NaturalesFísica, Profesor Titular Dpto de Física Universidad Francisco de Paula Santander, Investigador Asociado Grupo GITEC. Email: hjdulce@ufps.edu.co 


\section{INTRODUCCIÓN}

La descarga eléctrica se puede definir, en forma general, como la ionización inducida (generación de cargas o plasmas) y formación de corrientes (movimiento de cargas) por efecto de campos eléctricos y/o magnéticos. Entendiéndose el plasma como un gas ionizado con un comportamiento colectivo de la distribución de cargas.

Comúnmente se define la descarga eléctrica como el paso de corriente entre dos electrodos, esto sería solo para un caso particular de descarga, ya que no necesariamente se produce entre electrodos. Por lo anterior se hace necesario profundizar sobre los tipos de descargas.

\section{DESCARGAS ELÉCTRICAS}

Una primera clasificación, en función del medio a través del cual se produce la ruptura, permite dividir las descargas eléctricas en gases y descargas eléctricas en líquidos. Estas últimas poco estudiadas, pero con muchas aplicaciones como las mencionadas por Yang, Cho y Fridman (2012).

Descargas en Gases. Las descargas en gases han sido las más estudiadas experimentalmente; sin embargo, debido a las diferencias en su comportamiento, se debe hacer una clasificación en dos grandes grupos, las descargas a bajas presiones o en vacío (DBP) y las descargas a presión atmosférica (DPA). Por otra parte el potencial o voltaje permite dividir en dos grandes grupos descargas a bajos voltajes y de alto voltaje, es decir por debajo o por encima de unos 2.000 Voltios. Adicionalmente, dependiendo del tipo de fuente o alimentación tendremos las descargas de corriente directas (CD), de corriente alternas (CA), y las descargas pulsadas. Para éstas últimas resulta de gran importancia el rango de frecuencias en las cuales se trabaje, este puede ser bajas frecuencias (hasta el orden de kilohertz, $\mathrm{KHz}$ ), radiofrecuencia (del orden de megahertz, MHz), y microondas (del orden de gigahertz, GHz). Finalmente dependiendo del acoplamiento las descargas eléctricas pueden tener acoplamiento capacitivo (por ejemplo electrodos planos paralelos), inductivo (por ejemplo con bobinas como las antorchas plásmicas), o guía de ondas (por ejemplo microondas).

Para el caso de las descargas eléctricas de corriente continua, entre electrodos paralelos, se ha encontrado un comportamiento que permite relacionar el potencial de ruptura, es decir el voltaje al cual se inicia la descarga, con relación al producto de presión distancia entre electrodos; dicho comportamiento se conoce como la ley de Paschen, y se representa en la figura 1 conocida como curva de Paschen.

Los valores específicos, especialmente el potencial de ruptura mínimo, son propios de cada gas o mezcla de gases. Estudios realizados en las descargas pulsadas de alto voltaje a bajas presiones (Dulce, 2002) han demostrado que, en la rama izquierda de la curva de Paschen, el comportamiento es diferente, ya que para una distancia y presión fija existe un rango de valores de voltaje en el cual se da el encendido de la descarga.

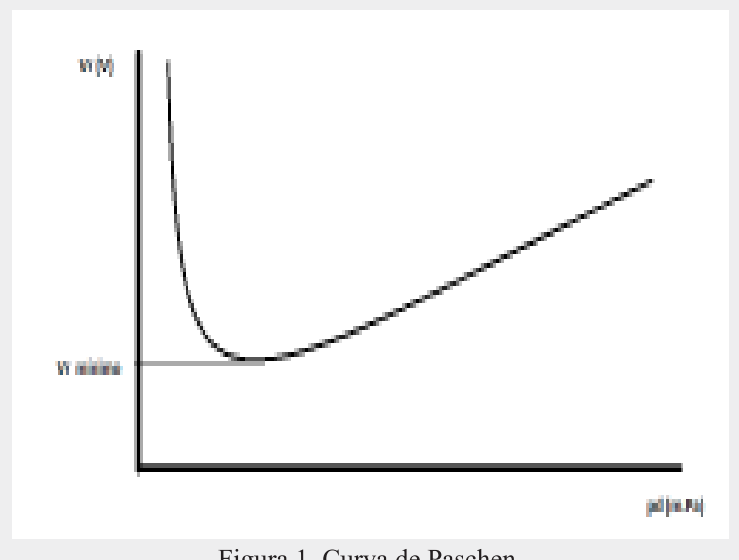

En la figura 2 se puede observar la diferencias en el comportamiento de las descargas eléctricas en vacío cuando suceden a bajos voltajes (menos de 2000 Voltios) y a alto voltaje. Uno de los primeros trabajos en el cual se analizó la característica voltio-ampérica (CVA) de las descargas de alto voltaje que encienden en la rama izquierda de la curva de Paschen es el trabajo de Druyvestein y Penning (1940). 
Eco.Mat Cúcuta-Colombia

Vol. 6

No. 1

Enero-Diciembre 2015 ISSN 1794-8231 ISSN 2462 - $8794 \mathrm{Web}$ PP: 6-21
Descargas eléctricas y sus aplicaciones

En la figura 2 se identifican cuatro zonas: Descarga oscura (dark), descarga luminiscente (glow) normal, descarga luminiscente anormal, y descarga de arco. Las descargas con mayor número de aplicaciones tecnológicas son en su orden las descargas luminiscentes anormales y luminiscentes normales, y las descargas de arco.

En la figura 2 se presenta una vista esquemática de la CVA de estas descargas y la comparación con la CVA de descargas en la rama derecha (según Druyvestein y Penning). En condiciones de presión muy baja, pd < $(\mathrm{pd})_{\min }$, (donde $\mathrm{p}$ es la presión del gas, $\mathrm{d}$ es la distancia interelectródica y (pd) ${ }_{\min }$ es el mínimo en la curva de Paschen) la descarga luminiscente normal no se enciende pues la anchura de la capa catódica es mayor que la distancia entre los electrodos. La descarga que aparece en este caso se caracteriza por un voltaje grande de encendido y por esta razón se denomina descarga luminiscente de alto voltaje. Esta forma de descarga se caracteriza por un desarrollo débil de cargas volumétricas ya que el aumento de la separación entre los electrodos se acompaña por la disminución del voltaje en la descarga.

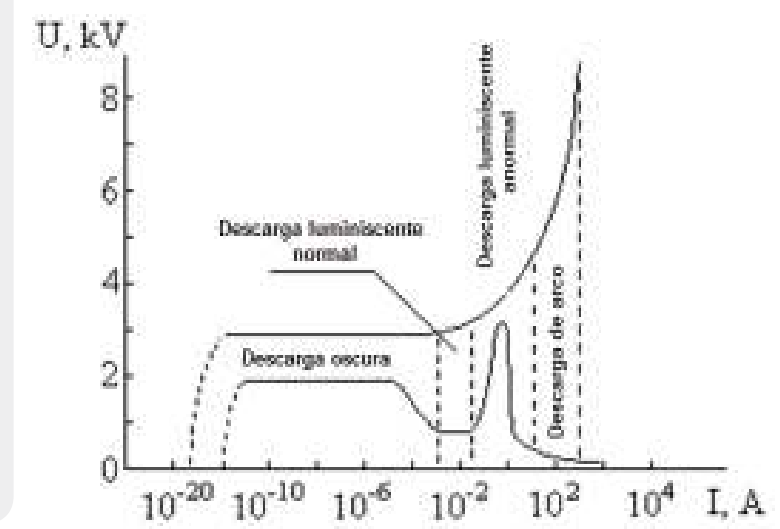

Figura 2. Característica volti-ampérica descargas corriente directa en vacío.

Fuente: Druyvesteyn M., Penning F., Rev. Mod. Phys., 12, (1940) 89-105.

Lo común a todas estas descargas son los procesos básicos que garantizan su formación y su estabilidad. Uno de los más importantes es la colisión entre partículas, como se mencionó al comienzo del capítulo, en las descargas se produce movimiento de partículas cargadas que interactúan con los átomos y/o moléculas del gas; dependiendo del tipo de colisión que se produzca se van a generar excitaciones, ionizaciones $\mathrm{y} / \mathrm{o}$ recombinaciones; cada una de las cuales se tratarán a profundidad más adelante. Esta visión microscópica de lo que acontece durante el fenómeno de las descargas eléctricas es lo que permite intuir que las descargas tienen diferentes tipos de comportamiento dependiendo de factores como la presión, la distancia entre los electrodos, el tipo de gas, la temperatura, la intensidad del campo eléctrico y/o magnético, la frecuencia de fluctuación de dichos campos, etc.

En el análisis de los procesos en una descarga incandescente de alto voltaje (DIAV) el espacio inter-electródico se trata como dos regiones conectadas en serie: la de caída catódica y la de plasma anódico (Novikov, 1983,46; Zavialov, Krendel, Novikov y Shanturin, 1989,58) (ver figura 3). La generación inicial de iones uni-cargados tiene lugar en la región del plasma anódico. Se supone que la frontera externa del plasma anódico con la densidad $\mathrm{n}$ sirve como una fuente de iones que se aceleran por el gradiente del campo eléctrico en la zona de caída catódica donde se produce prácticamente toda la caída del voltaje aplicado a la descarga. Un cuadro de distribución de las corrientes en el espacio de la descarga se da en la figura $3 \mathrm{~b}$.

La densidad de la corriente iónica a través de la frontera del plasma se determina por la relación conocida de Bohm (Huddlestone y Leonard, 1965, 150):

$$
j^{+} \approx 0,35 e \cdot n \sqrt{\frac{2 k T_{e}}{M^{+}}}
$$

donde e es la carga elemental, $n$ la densidad del plasma, $\mathrm{M}^{+}$la masa del ion, $\mathrm{k}$ la constante de Boltzman, $\mathrm{y}_{\mathrm{e}}$ la temperatura electrónica.

Dependiendo de las condiciones, en la cámara de vacío, para el flujo iónico se pueden presentar dos opciones: el caso a colisional de movimiento o el caso de movimiento con 
múltiples colisiones elásticas e inelásticas con los átomos del gas. En el caso del régimen de alto vacío (acolisional) la relación entre la corriente iónica sobre el cátodo $\mathrm{j}^{+}$, la caída catódica de potencial $\mathrm{U}_{0} \mathrm{y}$ las dimensiones de la zona de caída catódica $\mathrm{d}_{0}$ normalmente se describe por la ecuación de Child-Langmuir (Lieberman y Lichtenberg, 1994, 165):

$$
j^{+}=\frac{4}{9} \varepsilon_{0}\left(\frac{2 e}{M^{+}}\right)^{1 / 2} \frac{U_{0}^{3 / 2}}{d_{0}^{2}}
$$

La dependencia analítica para los modelos de régimen colisional del movimiento de iones, que se encuentra como solución de la ecuación de Poisson, contiene un factor que incluye el efecto de la presión del gas de trabajo p (Novikov, 1983, 96):

$j^{+}=\frac{4}{9} \varepsilon_{0}\left(\frac{2 e}{M^{+}}\right)^{1 / 2} \frac{U_{0}^{3 / 2}}{d_{0}^{2}}\left(1+0,319 \frac{d_{0}}{\lambda_{i-a}}\right)^{-1 / 2}$

Donde la longitud de carrera respecto al cambio de carga (recarga) $\lambda_{i-a}=1 / n_{a} \sigma_{i-a}$, es proporcional a $1 / \mathrm{p} \sigma_{\mathrm{i}-\mathrm{a}}$, . La relación entre las dimensiones de la zona de caída catódica y la presión de trabajo de la descarga $\mathrm{pd}_{0}=$ constante fue determinada experimentalmente por McClure $(1961,977)$.

Bajo la acción del flujo de iones y de partículas neutras incidentes al cátodo, a cuenta de los procesos de emisión ion-electrón, se forma un flujo electrónico desde el cátodo. La relación entre los dos flujos se determina por la característica electro-física del cátodo, el coeficiente de emisión electrónica secundaría $\gamma_{i-\mathrm{e}}$. En una descarga incandescente clásica existe el mismo mecanismo de emisión de electrones. La aceleración siguiente en la zona de la caída catódica lleva a una ionización intensa del gas por choques con electrones, a la creación del flujo iónico, y al paso hacia el régimen auto-sostenido de la descarga incandescente. En el caso de la descarga de alto voltaje, en la rama izquierda de la curva de Paschen, el cuadro cambia significativamente: la caída de voltaje en la región catódica alcanza decenas o centenas de kilo-voltios (aproximadamente $90 \%$ de $\mathrm{U}_{\mathrm{p}}$ ). Con todo eso, como lo mostraron muchos estudios, la caída principal de potencial se produce en una zona estrecha cerca del cátodo $\left(\mathrm{d}_{\mathrm{u}}\right)$.

Rigurosamente hablando la distribución del potencial, que es típica para la mayoría de descargas de alto voltaje en la rama izquierda de la curva de Paschen (la curva negra en la figura 3c), es bastante extraña. En realidad, ella no corresponde a la distribución en el diodo de vacío (a la solución de la ecuación de Poisson). La caída de potencial debería ser menos inclinada, con el gradiente del campo eléctricomás pequeño cercadel cátodo(la curva azul en la Figura 3c). Es decir la dependencia de la corriente con el voltaje no corresponde a la "ley $3 / 2$ ". Pero todos los argumentos obtenidos experimentalmente, incluso los experimentos descritos en este trabajo, están a favor de la distribución observada. Entonces todos los electrones de emisión se aceleran hasta energías aproximadamente igual a eU . Según McClure $(1961,979)$ la fracción de los electrones plásmicos "lentos" acelerados en la región de la caída catódica con el campo eléctrico moderado (fuera de los límites de la capa catódica estrecha $d_{u}$ ) no es grande y se estima no mayor al $10 \%$ de la corriente del haz electrónico.

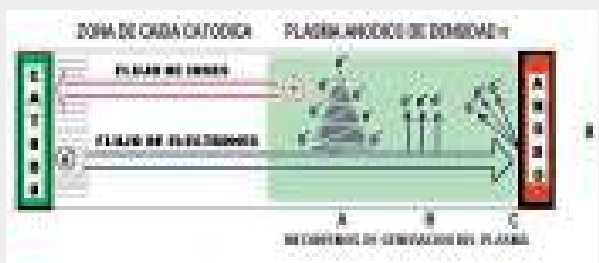

id
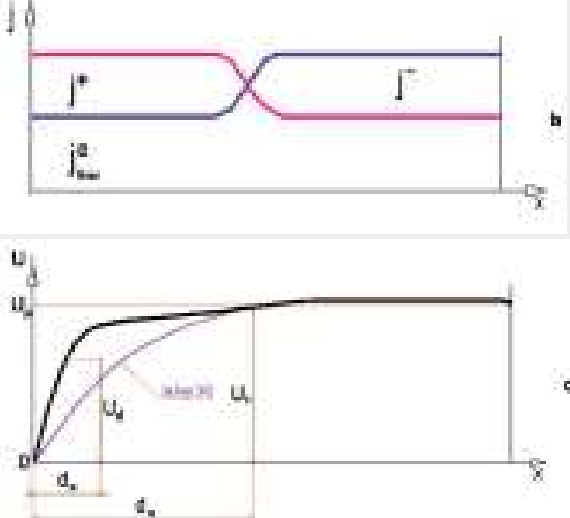

Figura 3. Esquema simple de un modelo de la descarga (a), distribución de la densidad de corriente (b) y del potencial (c)
Enero-Diciembre 2015 ISSN 1794-8231 ISSN 2462 - $8794 \mathrm{Web}$ PP: 6-21 
Eco.Mat

Cúcuta-Colombia

Vol. 6

No. 1

Enero-Diciembre 2015

ISSN 1794-8231

ISSN 2462 - $8794 \mathrm{We}$

PP: 6-21 descargas de alto voltaje en la rama izquierda de la curva de Paschen la longitud de la carrera libre de los electrones de emisión $\lambda$ es mucho mayor que las dimensiones del intervalo de descarga (ánodo-cátodo) $\mathrm{d}_{\mathrm{a}-\mathrm{c}} \mathrm{y}$ el haz catódico no puede participar directamente ni en el proceso de creación del flujo iónico hacia el cátodo ni en el de formación del plasma anódico. Por eso, es válida y muy actual, para esta familia de descargas, la pregunta sobre el mecanismo de generación del plasma de descarga con la densidad $\mathrm{n}$ suficiente para lograr el régimen auto-sostenido.

En trabajos publicados hasta los años 80, sobre el estudio de descargas de alto voltaje (McClure, 1961,982; Krendel, 1977, 124; Novikov, 1983, 56), se considera el mecanismo de ionización del gas de trabajo por electrones "reflejados" del ánodo (mecanismo C en la figura 3a) como uno de los más probables. El ánodo está bajo la acción de haces catódicos, de cuantos de rayos $\mathrm{X}$ de frenado $\mathrm{y}$ de un flujo iónico acelerado por la caída catódica. Bajo estos factores aparece la emisión electrónica. La parte de los electrones resultantes de los procesos de fotoemisión y emisión ión-electrón es pequeña, la mayor parte la aportan los electrones reflejados no elásticamente por el ánodo. El coeficiente de reflexión de electrones depende de: el ángulo de incidencia al ánodo, el material del ánodo, la energía de los electrones, si hay o no una película superficial en el ánodo etc. El estudio detallado y las estimaciones del balance de las partículas cargadas demostraron que tal mecanismo de generación garantiza la condición de régimen auto-sostenido de la descarga solo en un 10-50\% (Zavialov, Krendel, Navikov y Shanturin, 1989, 204). Finalmente, la influencia de la ionización por los electrones (distintos a los del haz catódico) provenientes de la región de la caída catódica y, en el caso típico de la descarga cilíndrica, la emisión electrónica secundaria de las paredes de cuarzo (mecanismo B en la Figura 3a) no produce un aporte significativo en el balance de las partículas cargadas (McClure, 1961, 204).

Aunque es evidente que los mecanismos
Descargas eléctricas y sus aplicaciones

estudiados son adecuados, y se pueden dar sin duda en la descarga, es muy probable que precisamente ellos sean los responsables de la aparición inicial del plasma en el espacio de la descarga. Sin embargo en el proceso de generación de partículas cargadas el papel dominante lo juegan los procesos de interacción colectiva "haz-plasma".

El mecanismo de haz de calentamiento de los electrones plásmicos es bien conocido (Levitsky y Shanturin, 1965, 1182; Levitsky y Shanturin, 1967, 350). La esencia de este mecanismo de generación está en que a cuenta de la interacción coulombiana, entre los electrones del haz y las cargas en el plasma, se excitan las oscilaciones intensas de Langmuir que producen calentamiento acolisional de los electrones plásmicos (Femsier, Manheiner, Meger y Mathew, 1998, 2140). En ciertas condiciones el calentamiento puede ser eficiente para la ionización intensa del gas que compensa totalmente la pérdida de las partículas cargadas y garantiza el régimen auto-sostenido de encendido de la descarga. Para las condiciones de existencia de la descarga de alto voltaje el mecanismo "haz-plasma" (mecanismo A en Fig. 3a) es el mecanismo más eficaz en la generación de partículas cargadas en el espacio de la descarga (Zavialov, Krendel, Navikov y Shanturin, 1989, 113). A continuación se realizaran las estimaciones respectivas de los mecanismos posibles de generación de las partículas cargadas en la región del plasma anódico para la descarga de estudio.

Descarga en Líquidos. El estudio de las descargas en líquidos nace de la necesidad de estudiar los procesos en el maquinado mediante descargas eléctricas (Electrical Discharge Machinining, EDM) y el tratamiento de aguas. En primera instancia se trataron de identificar como descargas de barrera dieléctrica, considerando como dieléctrico el líquido correspondiente, pero que posteriormente se demostró que la ruptura y participación de componentes del líquido en el plasma que se forma por la descarga lo hacen diferente (Yang Y., Cho Y.I., y Fridman A., 2012, 15). 
Aunque la teoría de la descargas es la misma que la de las descargas en gases, la formación de burbujas hace que se den particularidades en cuanto a la interacción de la radiación y de las partículas cargadas con los sustratos líquidos y/o sólidos.

\section{FÍSICA DEL PLASMA}

Se conoce como plasma a un medio gaseoso que contiene un número apreciable de cargas libres pero que es aproximadamente neutro en su conjunto. La gran cantidad de cargas libres generan altas conductividades eléctricas, las que pueden establecer fácilmente corrientes que interactúan con campos magnéticos aplicados y con los propios producidos por tales corrientes eléctricas. Un porcentaje mayor al $99 \%$ de la materia en el universo existe en forma de plasma; el medio estelar, interplanetario e interestelar, y las altas atmósferas planetarias (ionosferas). Sin embargo, en los medios relativamente densos y/o fríos en los que se desarrolla la vida el estado de plasma es más raro por la tendencia a la recombinación de las cargas libres. Experimentalmente se debe suministrar energía a un gas para producir el estado de plasma, y para mantenerlo cuasi-estable, sobre todo en las condiciones necesarias para algunas aplicaciones específicas.

\section{Parámetros de un Plasma}

Los plasmas pueden caracterizarse a partir de sus temperaturas, diferenciando las de las partículas neutras de las iónicas y electrónicas por una parte, y por otra por las concentraciones de las diferentes especies. Sin embargo los parámetros que más influyen en la estabilidad de los plasmas son la longitud de Debye, la cuasi-neutralidad, y el comportamiento colectivo.

Longitud de Debye. Si le entregamos energía a un gas, comúnmente produciendo una descarga eléctrica a través de éste, los electrones emitidos por el cátodo producirán disociación e ionización de las moléculas del gas, aumentándose el número de electrones capaces de ionizar. Por otra parte, los electrones libres emiten radiación electromagnética al ser acelerados en su interacción con otras partículas, y los iones, átomos y moléculas emiten y absorben radiación al des-excitarse o excitarse a distintos niveles; esta radiación también produce ionizaciones. Se establece eventualmente un equilibrio entre los distintos tipos de ionizaciones, recombinaciones y pérdidas y ganancias de partículas a través del contorno, sostenido por el circuito externo. En este estado de plasma tendremos una población de electrones, iones con distintos estados de carga y excitación, partículas neutras (moléculas y átomos) también en distintos estados excitados, y radiación electromagnética. La población relativa de cada una de estas especies dependerá del tipo de equilibrio que se establezca; los procesos de ionización más importantes son los debidos a impacto electrónico (e $+\mathrm{A}$ $\rightarrow \mathrm{A}^{+}+2 \mathrm{e}$ ) y la conocida como ionización por radiación ( $\left.\mathrm{hn}+\mathrm{A} \rightarrow \mathrm{A}^{+}+\mathrm{e}\right)$, mientras que las recombinaciones corresponden a los procesos inversos; recombinación a tres cuerpos $\left(\mathrm{A}^{+}+2 \mathrm{e} \rightarrow \mathrm{e}+\mathrm{A}\right)$ y recombinación con emisión de radiación $\left(\mathrm{A}^{+}+\mathrm{e} \rightarrow \mathrm{hn}+\mathrm{A}\right)$. En equilibrio termodinámico estos cuatro procesos estarían equilibrados; por lo tanto habría una temperatura única para todas las especies, a la que cada proceso y su inverso producirían la misma cantidad de reacciones por unidad de tiempo, lo que requiere una población relativa de especies muy particular para cada temperatura. Muy rara vez el plasma es lo suficientemente extenso y denso para retener la radiación y establecer así un equilibrio con ella; de todas maneras es todavía posible tener equilibrio termodinámico entre los procesos que no involucran radiación; la ecuación de Saha permite entonces obtener la población relativa de especies. Sin embargo, a las densidades y temperaturas habituales de plasmas de laboratorio la recombinación a tres cuerpos es generalmente mucho menos probable que la recombinación radiactiva. El resultado es entonces que muchas veces se establece un equilibrio de poblaciones no termodinámico, en el que la ionización por impacto es balanceada por recombinación radiactiva.

Los procesos inelásticos considerados 
Eco.Mat Cúcuta-Colombia Vol. 6 No. 1 Enero-Diciembre 2015 ISSN 1794-8231 ISSN 2462 - $8794 \mathrm{Web}$ PP: 6-21
Descargas eléctricas y sus aplicaciones

producen entonces un cierto nivel de población de las especies del plasma. Estas especies interactúan también a través de procesos elásticos, especialmente las especies cargadas a través de la fuerza eléctrica de largo alcance.

Aunque el alcance de la fuerza eléctrica entre dos cargas en el espacio vacío es infinito, en el interior del plasma éste se reduce debido a la presencia de las demás cargas de signo contrario que rodean cualquier carga. Imaginemos un ion positivo en un plasma debido a la fuerza de Coulomb, toda una nube de electrones negativos será atraída hacia ese ion. Estos electrones formarán alrededor del ion una coraza de carga contraria que impedirá que la carga de este ion sea sentida por cargas más lejanas. Lo mismo ocurrirá con los electrones y de esta manera se genera un efecto de apantallamiento, que limita el alcance real de la atracción o repulsión de cada carga hacia sus vecinas. Esta distancia, que podríamos definir como el radio de la esfera real de influencia de cada carga dentro del plasma se conoce como la distancia de Debye o la longitud de Debye. Debe su nombre al físico holandés Peter Debye, quien fue el primero en reconocer este efecto para aplicar el movimiento de los iones en la solución de un electrolito. Esta distancia está dada por (Harry J.E., 2010, 36):

$\lambda_{\mathrm{D}}=\left[\left(\varepsilon_{0} \mathrm{kT}_{\mathrm{e}}\right) /\left(\mathrm{n}_{\mathrm{e}} \mathrm{e}^{2}\right)\right]^{1 / 2}$

donde $\mathrm{k}$ es una constante de Boltzmann (1.38 $\left.\mathrm{x} 10^{-23} \mathrm{Joule} / \mathrm{K}\right), \mathrm{T}$ es la temperatura absoluta en Kelvin), $\varepsilon_{0}$ es la permitividad del espacio libre $\left(8,85 \times 10^{-12} \mathrm{C}^{2} / \mathrm{N} \mathrm{m}^{2}\right), \mathrm{n}_{\mathrm{e}}$ es el número de cargas (positivas o negativas) por metro cúbico y e es la carga del electrón $(1.60 \mathrm{x}$ $10^{-19} \mathrm{C}$ ). Esto puede expresarse en el sistema M.K.S. (Harry J.E., 2010, 37), es decir calculada en metros como:

$\lambda_{\mathrm{D}}=69 \times\left(\mathrm{T}_{\mathrm{e}} / \mathrm{n}_{\mathrm{e}}\right)^{1 / 2}$

De esta manera, la distancia de Debye crece al aumentar la temperatura, pues en los gases más calientes las partículas se mueven más rápidamente $\mathrm{y}$ entonces el apantallamiento es menos efectivo; y disminuye al aumentar la densidad de las cargas, pues cuando hay una gran densidad de cargas el apantallamiento es mayor. Es importante hacer notar que es precisamente la temperatura (el movimiento agitado de los elementos del plasma) la que impide que las cargas se recombinen para formar átomos o moléculas neutras. Los plasmas fríos sólo pueden mantenerse a muy baja densidad, como los interestelares e intergalácticos, ya que en ese caso tampoco es muy probable que haya recombinaciones. Aunque no se ha demostrado que esta relación pueda aplicarse en el interior de un gas ionizado, la distancia de Debye es un buen indicador de la distancia a la cual domina la influencia de cada partícula.

Como cada partícula tiene su propia esfera de Debye, es de esperar que estas esferas se traslapen y de esta manera el plasma va a responder colectivamente. Como ya se mencionó, la distancia de Debye aumenta al disminuir la densidad; sin embargo, si la densidad de carga en un gas ionizado es $\tan$ baja que no hay suficientes cargas en las dimensiones del plasma como para garantizar un comportamiento colectivo, este gas ionizado no será un plasma. Es fácil ver que en este caso tampoco podrá obtenerse la condición de cuasi-neutralidad. De hecho, para que un plasma exista es necesario que las dimensiones del espacio ocupado por el plasma sean mucho mayores que la longitud de Debye. Por ejemplo, en el medio interplanetario, la densidad de electrones (y de iones) es de 10 por centímetro cúbico. En las dimensiones espaciales este medio se comporta como un plasma y presenta toda la gama de interesantes procesos que caracterizan a los plasmas. Pero si en una botella de laboratorio colocamos un gas formado por iones y electrones con una densidad de 10 por $\mathrm{cm}^{3}$, tendríamos un vacío prácticamente perfecto del cual no podríamos obtener ninguna respuesta. Ésta representa una de las dificultades para experimentar con plasmas pues no son escalables, y en general los prototipos de laboratorio no se comportarán como los enormes sistemas naturales.

La cuasi-neutralidad. Puesto que en el plasma 
existe muy poca restricción al movimiento de las cargas, tiende a mantener un estado de neutralidad eléctrica aún en regiones muy pequeñas. Cualquier acumulación de carga de un solo signo en cierta región del plasma producirá una fuerza atractiva para las cargas opuestas lo suficientemente grande como para recuperar el equilibrio de la carga eléctrica casi instantáneamente. De esta manera, si el plasma no es forzado por campos eléctricos o magnéticos muy intensos a mantener acumulaciones locales de carga, permanecerá en un estado de cuasi-neutralidad; esto es, un estado en el que la concentración de cargas positivas será prácticamente igual a la concentración de cargas negativas, aun en pequeñas regiones del espacio.

Los movimientos colectivos y la cuasineutralidad son fundamentales para el estado de plasma, al grado que suele definirse al plasma como un sistema cuasi-neutro constituido por un gran número de partículas cargadas que exhiben movimientos colectivos.

Comportamiento colectivo. Una de las principales características de la materia en estado de plasma es su capacidad de responder colectivamente a impulsos internos y externos. Este comportamiento fue descrito por primera vez por John Willian Strutt Rayleigh. Los movimientos colectivos del plasma son consecuencia del gran alcance de las fuerzas electrostáticas que sienten entre sí las partículas cargadas que lo componen. A la fuerza entre dos partículas cargadas se le conoce como fuerza de Coulomb, porque fue el físico francés Charles Charles-Augustin Coulomb quien midió por primera vez la fuerza entre cargas eléctricas a diferentes distancias. Según la relación encontrada por Coulomb:

$\mathrm{F}_{\mathrm{e}}=\mathrm{K}_{\mathrm{e}} \mathrm{q}_{1} \mathrm{q}_{2} / \mathrm{r}^{2}$

Lo que indica que la fuerza $F_{e}$ depende directamente del producto de la magnitud de las cargas $\left(\mathrm{q}_{1}\right.$ y $\left.\mathrm{q}_{2}\right)$ de las partículas que interactúan e inversamente del cuadrado de la distancia (r) que las separa, donde $\mathrm{K}_{\mathrm{e}}$ es la constante electrostática $\left(\mathrm{Ke}=8,99 \times 10^{-9} \mathrm{~N} . \mathrm{m} /\right.$ $\mathrm{C}^{2}$ ). Esta fuerza se incrementa al aumentar la carga de cualquiera de las partículas y disminuye al aumentar la distancia entre ellas. Sin embargo, aunque la fuerza disminuye con la distancia, para que se reduzca a cero, es necesario que la distancia entre las cargas sea infinita. De esta manera, una carga eléctrica sentirá la presencia de otra a distancias muy grandes.

De la experimentación con cuerpos cargados eléctricamente se sabe que las cargas del mismo signo (positivas con positivas o negativas con negativas) se repelen, mientras que las de signos diferentes se atraen. La fuerza entre cargas eléctricas es muy semejante a la fuerza gravitacional entre dos cuerpos masivos, pero en este último caso la fuerza entre dos masas siempre es de atracción, mientras que entre cargas la fuerza puede ser de atracción o de repulsión, dependiendo del signo de la carga.

Las fuerzas eléctricas entre partículas son mucho mayores que sus fuerzas de atracción gravitacional. Por ejemplo, entre un protón y un electrón a una distancia cualquiera, la fuerza de atracción eléctrica es 1039 veces mayor que la fuerza de atracción gravitacional. De esta manera, las fuerzas dominantes en el interior del plasma son las eléctricas, las cuales, como ya dijimos, son de muy largo alcance. En el interior de un gas compuesto por átomos o moléculas neutras, las interacciones entre sus componentes sólo se darán a distancias comparables a las dimensiones geométricas de los mismos. Pero en el interior de un plasma las partículas interactúan a distancias mayores y de esta manera, aun los plasmas de muy baja densidad funcionan de manera cohesiva. Cada ion y cada electrón en el interior de un plasma puede sentir la influencia de muchas partículas a su alrededor, de manera que el comportamiento del plasma estará determinado por interacciones colectivas y no solamente por la interacción entre dos partículas individuales.

\section{APLICACIONES DE LAS DESCARGAS ELÉCTRICAS}

Las aplicaciones de las descargas se clasificaran en tres grandes grupos: En el primero las aplicaciones relacionadas con el 
Eco.Mat Cúcuta-Colombia

Vol. 6

No. 1

Enero-Diciembre 2015 ISSN 1794-8231

ISSN 2462 - $8794 \mathrm{Web}$ PP: 6-21

14
Descargas eléctricas y sus aplicaciones

tratamiento superficial, dentro de las cuales está el maquinado con descargas eléctricas, los recubrimientos duros entre otros; el segundo grupo formado por las aplicaciones en medicina, ambientales y biotecnológicas; y un tercer grupo aplicaciones de tipo nanotecnológico.

\section{Tratamiento Superficial}

Uno de los problemas de más actualidad en la industria metalmecánica de alta tecnología y en la fabricación de aparatos de precisión es el desarrollo de técnicas de tratamiento superficial avanzado que logren mejorar propiedades de los materiales tales como: microdureza, resistencia al desgaste por fricción, resistencia a la corrosión, etc. No hay duda que el progreso en estas ramas está ligado ante todo con los éxitos en el diseño y construcción de dispositivos que, como resultado de amplios estudios y del control de los procesos que en ellos se suceden, logren la producción de materiales con propiedades tribológicas predeterminadas de acuerdo con una necesidad específica.
Desde los años 70 se realizan investigaciones intensas en el área de tratamiento de superficies de materiales de construcción por haces de partículas cargadas y especialmente por haces iónicos.

Dependiendo de la energía con la que los iones llegan a una superficie puede prevalecer uno de tres procesos diferentes (ver figura 4). Si la energía es muy baja (hasta decenas de electrón-voltios) los iones se depositan en la superficie, creándose un recubrimiento - película delgada. Si la energía es mayor (algunas centenas de electrón-voltios) predomina un proceso de chisporroteo de la superficie (conocido como sputtering), dicho proceso es útil para limpieza superficial o para evaporar materiales en procesos de recubrimiento. Finalmente, a energías mucho mayores (desde 5 kilo-electrón-voltios hasta algunos Mega-electrón-voltios), los iones penetran dentro de la superficie sólida incrustándose a una profundidad de varias capas atómicas. Este proceso se conoce como implantación iónica.
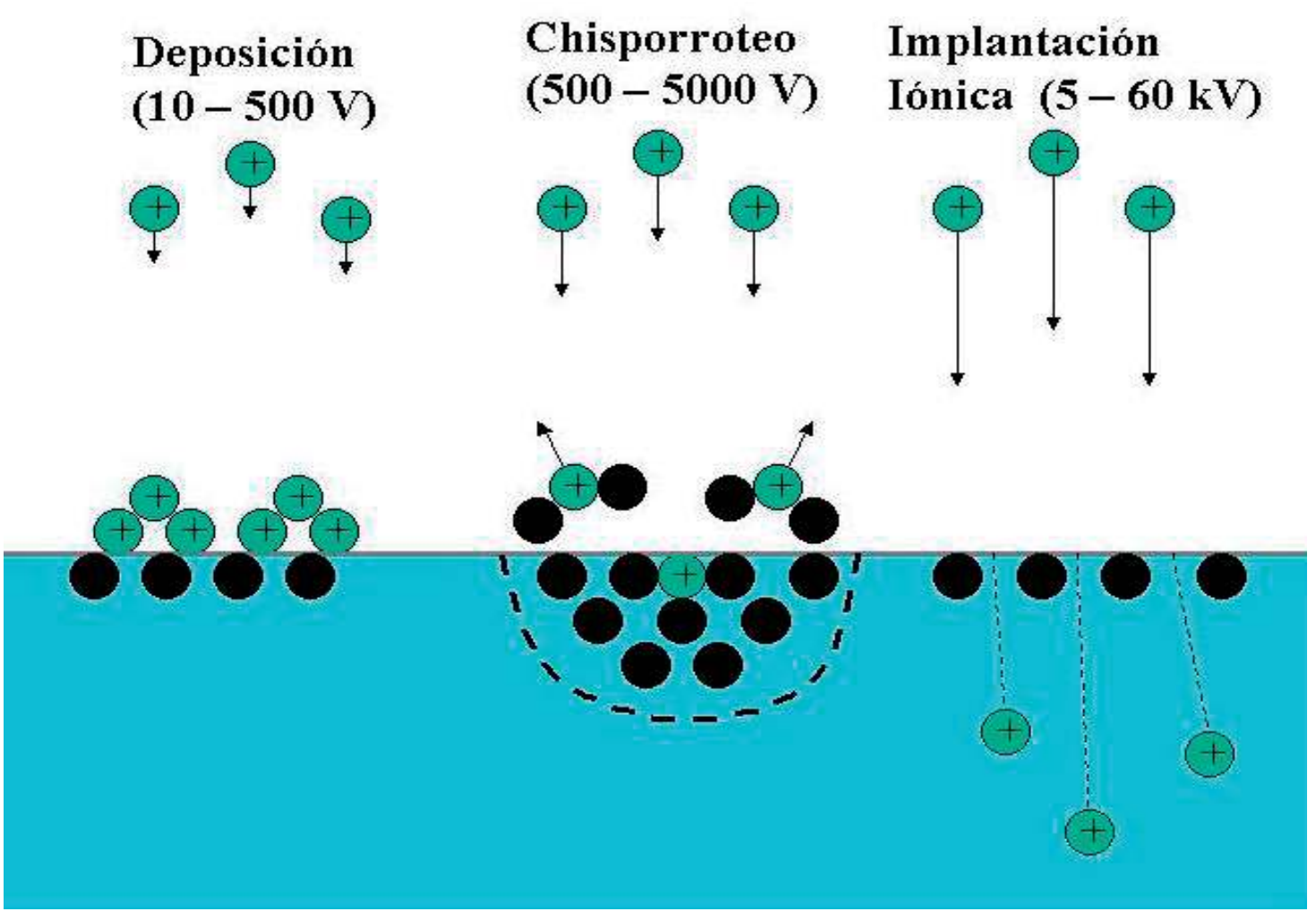

Figura 4. Tipos de procesos según el potencial acelerador. 
Las tecnologías iónicas se refieren por su realización técnica a métodos vacío-plásmicos que permiten producir capas modificadas con adhesión del $100 \%$ al material tratado diferenciándose y superando en este aspecto a los métodos tradicionales de aplicación de recubrimientos en vacío (magnetrón, acelerador, chisporroteo resistivo o activo).

En el proceso de implantación de iones acelerados tiene lugar la modificación en las capas superficial y subsuperficial del material original sin cambio en las dimensiones geométricas de la pieza tratada, y no se presenta como en el caso de chisporroteo una frontera bien definida capa - material original con diferentes características físicas (por ejemplo, el coeficiente de expansión térmica). En este sentido este método es próximo a los procesos tradicionales de saturación de superficies de materiales sólidos con adición de aleado tales como nitruración, carbonización, cementación con boro etc. Es muy importante tener en cuenta que el mecanismo de adición difiere radicalmente ya que la concentración de aditivo se determina no por las constantes de difusión del proceso en equilibrio de saturación de la superficie sino por las interacciones cinéticas del ion, acelerado hasta decenas kilo-electrón-voltios, con la red cristalina del material a tratar. Una consecuencia de esta diferencia es que la concentración del elemento implantado puede alcanzar un 20-70\% en comparación con 3-7\% que es común para los procesos de difusión. Con todo esto el tratamiento por implantación no se acompaña porcalentamiento significativo del material tratado que es una ventaja muy grande frente a los métodos físico-químicos donde la temperatura del procesamiento es alta, en algunos casos supera la temperatura de transformación de fase. Una temperatura alta puede producir deformación de piezas tratadas.

Después de los primeros experimentos sobre modificación de superficie por haces iónicos de alta energía se encuentra claro que es necesario estudiar los procesos de interacción de una partícula acelerada con el material bombardeado ya que el mecanismo de fortalecimiento de material es bastante complejo y depende de múltiples factores como del tipo de ion, su energía, dosis, composición química del material origen y temperatura entre otros. El ion frenado en el material participa y estimula una gran cantidad de procesos a nivel superficial y subsuperficial: crecimiento y traslación de dislocaciones, formación de nuevos compuestos químicos, destrucción de composiciones originales, difusión estimulada por radiación, etc., y el producto final del tratamiento es una resultante de todos estos procesos. Por lo anterior hasta la presente no se ha elaborado una teoría completa que abarque y determine en su totalidad el proceso de modificación de una superficie por un haz iónico.

Entrelos principales mecanismos queproducen endurecimiento de metal se pueden mencionar algunos: creación de composiciones químicas, defectos por radiación de la red cristalina, formación de capas subsuperficiales que provocan la tensión compresión - extensión en la superficie de material. Todavía no existe una solución del problema de determinar con exactitud cuál es el efecto de modificación y el comportamiento de la capa que se desgasta ya que el grosor de capas obtenidas por implantación iónica en la mayoría de los casos no supera un micrómetro $(1 \mu \mathrm{m})$. Sin embargo los resultados impresionantes en la modificación de propiedades tribológicas de materiales por los métodos de implantación iónica han obligado a los investigadores a buscar los métodos óptimos de la nueva tecnología, y a diseñar equipos con tecnologías especializadas. Una razón por la que no se utiliza ampliamente en la industria este método es la no existencia de un equipo simple, seguro y económico.

\section{Deposición de películas}

En el caso de la deposición de material utilizando descargas eléctricas, y por ende de plasmas, se encuentran dos técnicas muy utilizadas están son la de deposición por chisporroteo y deposición química de vapor (Chemical Vapor Deposition, CVD), que para el caso de la utilización de un plasma 
Eco.Mat Cúcuta-Colombia

Vol. 6

No. 1

Enero-Diciembre 2015 ISSN 1794-8231

ISSN 2462 - $8794 \mathrm{Wet}$ PP: 6-21 se denomina mejoramiento vía plasma de deposición química de vapor (plasma enhance Chemical vapor deposition, PE-CVD) (Bogaerts, Neyts, Gijbels, y van der Mullen, 2002, 630).

Un caso interesante relacionado con la deposición asistida con plasma es el descrito por Lundin y Pedersen (2013), para el endurecimiento de superficies cerámicas mediante la deposición de películas de cobre, titanio y carbón a una temperatura de $560^{\circ} \mathrm{C}$, mucho menor que la requerida en un termorociado.

La técnica de PE-CVD fue también utilizada por Grotjahn y otros (2013), para depositar películas de silicio que contenían carbono tipo diamante, mediante este estudio fue posible determinar la importancia del manejo de los parámetros de la descarga para poder obtener resultados óptimos en la formación de películas con determinadas características.

\section{Grabado (Etching).}

Mediante esta técnica se desprende material siguiendo un cierta uniformidad, comúnmente se utilizan descargas incandescente de corriente directa (dc) o de radiofrecuencia (rf) (Bogaerts, Neyts, Gijbels, y van der Mullen, 2002, 631).

\section{Maquinado mediante descargas eléctricas (EDM).}

Una de las aplicaciones de mayor desarrollo en los últimos años corresponde al maquinado mediante descarga eléctrica (electrical discharge machining, EDM), esta técnica se basa en los efectos erosivos que produce la energía termoeléctrica que circula entre la pieza de trabajo y un electrodo; el comportamiento del flujo de calor fue estudiado por Zhang $\mathrm{y}$ otros (2013) quienes concluyeron que el modelo de fuente de calor de Gauss es el que más se ajusta al EDM. El pulso de la descarga se genera a través de una pequeña distancia produciendo el desprendimiento del material no deseado mediante la fusión y vaporización de dicho material, una descripción completa de los procesos se dan el maquinado con
Descargas eléctricas y sus aplicaciones

descargas eléctricas es realizada por Khatter (2010), donde no solo hace una revisión de la literatura existente sino también propone un modelo térmico con los cálculos energéticos correspondientes.

Generalmente se utiliza como cátodo el electrodo del equipo y como ánodo la pieza de trabajo. Una revisión de las investigaciones sobre el EDM fue realizada por Abbas, Solomon y Bahari (2007); en dicho trabajo se describe los efectos de las vibraciones ultrasónicas asociadas cuando los pulsos de las descargas se encuentran dentro del rango ultrasónico (UEDM); de igual manera se discute la conveniencia del maquinado en seco (DEDM, Dry Electrical discharge Machining), teniendo en cuenta las ventajas comparativas desde el punto de vista ambiental, al no producirse vapores contaminantes que generan polución, a diferencia cuando el maquinado se efectúa en un medio líquido. Por otra parte Roth, Kuster y Wegener (2013) han realizado estudios sobre el efecto de la presencia de gases oxidantes en la estabilidad de la DEDM. De igual manera se proponen técnicas novedosas como el caso de la mezcla de polvos en las soluciones que se utilizan para el maquinado (powder mixed electrical discharge machining, PMEDM). Finalmente se realiza una descripción de las aplicaciones del maquinado en agua y se evalúan algunos modelos teóricos. Una revisión más completa y más reciente sobre el EDM fue realizada por Kumar, Singh, Singh, y Sethi (2009). En dicho trabajo se definen los parámetros asociados a los procesos del maquinado mediante descargas eléctricas, entre ellos: el voltaje, el pico de corriente, duración e intervalo de los pulsos, forma del pulso, polaridad, distancia inter-electródica, tipo de enjuague dieléctrico. Dichos factores pueden ser expresado en tres parámetros básicos: el llamado factor de trabajo, es decir el porcentaje de duración del pulso con respecto al tiempo total del ciclo; la frecuencia del pulso; y el promedio de corriente. Posteriormente se discute los efectos del maquinado sobre la capa superficial de los materiales, y las modificaciones superficiales generadas por la aplicación del EDM. De igual forma Weir y Pantoja (2012) realizaron 
un estudio de correlación entre la sensibilidad de las descargas eléctricas, la conductividad eléctrica y el tamaño de las partículas para el caso de materiales eléctricos compuestos.

Por otra parte el desarrollo de modelos teóricos del funcionamiento del EDM ha permitido la optimización de los procesos. No solo para proponer mejoras a la técnica, sino también para la implementación de equipos más robustos y con alternativas de aplicación (Joshi, y Pande, 2011). Un estudio de modelación y simulación de los procesos que ocurren en el EDM, con toda su complejidad, fue desarrollado por Weingärtner, Kuster, y Wegener (2012).

La industria en Malasia viene aplicando algunos cambios en la técnica EDM para convertirla en una manufactura más ecológica, combinando el maquinado con la destilación de agua, la cual sería utilizada como material dieléctrico (Abbas, Yusoff, y Mahmod@ Wahab, 2012).

Un estudio más profundo de modelización, para el caso de electrodos de carburo de tungsteno, con el propósito de optimizar el EDM fue realizado por Singh y Shukla (2012), en dicho estudio se tuvo en cuenta la geometría del cráter, la proporción de desgaste relativo y la rugosidad de la superficie adicionalmente a los parámetros tradicionales de EDM. Una de las conclusiones hace referencia a la baja eficiencia en la transferencia de energía, ya que tan sólo entre el $6,5 \%$ al $17,7 \%$ de la energía suministrada se utilizada efectivamente en el procesos de erosión, siendo ésta significativamente menor a la predicha por la modelización. Dicha eficiencia varía proporcionalmente con la corriente y la duración de los pulsos. Los valores óptimos son: $24 \mathrm{~A}$ de corriente, y entre 50 microsegundos y 200 microsegundos de duración de los pulsos.

Las aplicaciones del EDM han llegado incluso al campo de la investigación médica, un ejemplo de ello es el estudio realizado por Klocke, Schwade, Klink, Veselovac y Kopp (2013), su trabajo fundamentalmente se refiere a la compatibilidad de aleaciones con magnesio biodegradable, cuando las piezas para implantes ortopédicas son maquinadas mediante la técnica de EDM combinada con la conversión electrolítica vía plasma. Lamentablemente una de las conclusiones de dicha investigación se refiere a cierta toxicidad que se manifiesta mediante el ensayo de toxicidad in-vitro, posiblemente por la contaminación durante el proceso de maquinado con EDM, por lo cual se sugiere nuevos estudios con el propósito de determinar la causa de dicho resultado. Un trabajo realizado con el objeto de minimizar los efectos contaminantes en el EDM, a nivel microscópico, aplicable también al llamado maquinado electroquímico (ECM) y más específicamente al maquinado microelectroquímico pulsado ( $\mu \mathrm{PECM})$; la conclusión más importante de dicho estudio hace referencia al efecto que produce la presencia de contaminantes en la reducción del espacio entre el electrodo y la pieza de trabajo generando inestabilidades de las descargas y la formación de especies gaseosas no deseadas en el dieléctrico líquido utilizado (Schulze, y Shätzing, 2013).

Demellayer y Richard (2013) evaluaron la posibilidad de utilizar técnica del EDM para el maquinado de los llamados metales refractarios (Molibdeno y tungsteno), los cuales son difíciles de maquinar. Se encontró que el maquinado con EDM usando electrodos de filamento no dio los resultados esperados, ya que se consiguió una rugosidad de 0,22 micras que es cuatro veces mayor a la requerida, del orden de 0,05 micras, para las aplicaciones en el acelerador del CERN (centro Europeo de investigaciones nucleares), adicionalmente y como consecuencia de las tensiones residuales se presentan agrietamientos no deseados. Una aplicación similar para el caso del maquinado de mono-cristales de carburo de silicio fue estudiada por Zhao, Kuneida y Abe (2013). La conclusión de dicho trabajo de investigación es que la técnica de EDM tiene potencial aplicación en el rebanado o corte de obleas de carburo de silicio, aunque también observaron la formación de grietas resultado de las tensiones residuales del tratamiento. 
Eco.Mat Cúcuta-Colombia

Vol. 6

No. 1

Enero-Diciembre 2015 ISSN 1794-8231

ISSN 2462 - $8794 \mathrm{Web}$ PP: 6-21

18
Descargas eléctricas y sus aplicaciones

Assarzadeh y Ghoreishi desarrollan un estudio muy interesante en el que evalúan los resultados experimentales con respecto al modelo estadístico teórico del maquinado con EDM de un material compuesto, en este caso de carburo de cobalto- tungsteno $(\mathrm{WC} / 6 \% \mathrm{Co})$. Como conclusión el porcentaje de error no supera el $10 \%$, que para este tipo de procesos se puede considerar aceptable. Estudios similares se realizaron en la modificación de superficie de grafito mediante la EDM, en este caso se realizaron deposiciones de tungsteno, cromo, níquel y silicio sobre carbón y se analizaron las aleaciones que se obtienen por este método (Devarani, y Patowari, 2013).

Como se mencionó el maquinado de materiales cerámicos es difícil de realizar con otros métodos, al respecto Khanra y Pathak $(2012,7)$ realizan un estudio sobre el uso del EDM en el caso de los cerámicos avanzados especialmente en los buenos conductores como son: FeAl, Ni3Al, TiB2, y B4C.

Finalmente un estudio profundo del uso de la descargas eléctricas en el maquinado de hierro dúctil austemperizado fue realizado por Singh (2012).

\section{Implantación iónica}

En el proceso de implantación de iones acelerados tiene lugar la modificación en las capas superficial y subsuperficial del material original sin cambio en las dimensiones geométricas de la pieza tratada, y no se presenta como en el caso de chisporroteo una frontera bien definida capa - material original con diferentes características físicas (por ejemplo, el coeficiente de expansión térmica). En este sentido este método es próximo a los procesos tradicionales de saturación de superficies de materiales sólidos con adición de aleado tales como nitruración, carbonización, cementación con boro etc. Es muy importante tener en cuenta que el mecanismo de adición difiere radicalmente ya que la concentración de aditivo se determina no por las constantes de difusión del proceso en equilibrio de saturación de la superficie sino por las interacciones cinéticas del ion, acelerado hasta decenas kilo-electrón-voltios, con la red cristalina del material a tratar. Una consecuencia de esta diferencia es que la concentración del elemento implantado puede alcanzar un $20-70 \%$ en comparación con 3-7\% que es común para los procesos de difusión. Con todo esto el tratamiento por implantación no se acompaña por calentamiento significativo del material tratado que es una ventaja muy grande frente a los métodos físico-químicos donde la temperatura del procesamiento es alta, en algunos casos supera la temperatura de transformación de fase. Una temperatura alta puede producir deformación de piezas tratadas (Dulce, 2002, 14).
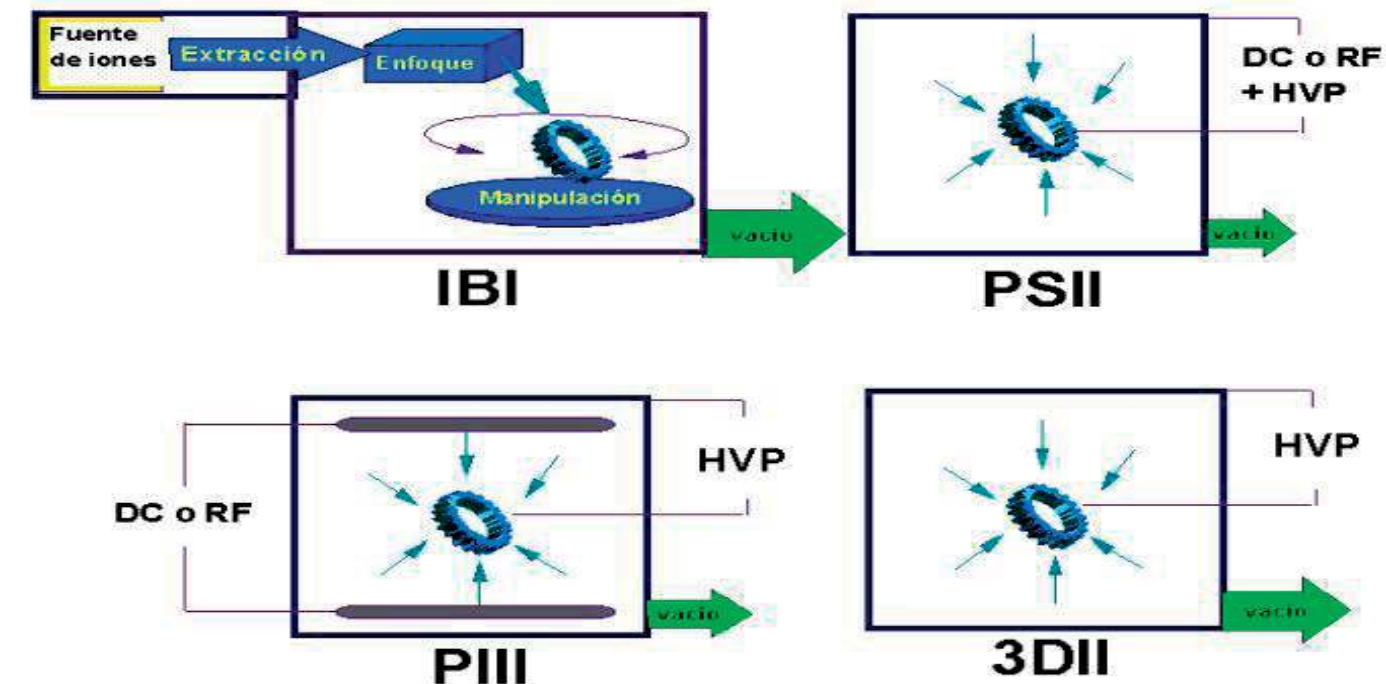

Figura 5. Evolución de las técnicas de implantación iónica 
En la figura 5 se presenta un esquema comparativo donde se puede observar el desarrollo de las técnicas de implantación: se inicia con la implantación por haces iónicos (IBI), que requieren montajes complejos y sistemas de ultra alto vacío (UHV). Se pasa por las técnicas tradicionales de implantación con base en la tecnología de los plasmas (PSII y PIII), que trabajan en un régimen de bajo vacío (LV) y que utilizan una combinación de fuentes DC o RF, para generar un plasma previo, y fuentes pulsadas de alto voltaje (HVP) con pulsos de muy corta duración (del orden de micro-segundos), para atraer los iones hacia las superficies a tratar. Finalmente se llega a la tecnología de Implantación Iónica Tridimensional (3DII), que trabaja en un régimen de alto vacío (HV) y que utiliza descargas pulsadas de alto voltaje (HVP) con pulsos rectangulares de duración hasta 2,5 mili-segundos, los cuales realizan simultáneamente las dos funciones requeridas para una implantación iónica eficiente, es decir generan un plasma de alta densidad $\mathrm{y}$ atraen los iones hacia la superficie de las muestras que funcionan como cátodo, sin que se presente movilidad de la capa catódica.

\section{Aplicaciones en Medicina y Ambientales}

Las descargas de corona, las de barrera dieléctricas son las más utilizadas en las aplicaciones en medicina y el campo de las ciencias medio ambientales, esto debido a la combinación de los procesos de corrientes electrónicas con fluyo de rayos ultravioleta. Estas aplicaciones van desde las cauterizaciones en microcirugía hasta el tratamiento de aguas a gran escala, de hecho los ozonizadores utilizan descargas para purificar el agua (Yang, Cho, y Fridman, 2012).

Un estudio muy interesante es la eliminación de residuos plásticos usando pirolisis vía plasma (Puncochar, Ruj, y Chatterjee, 2012), al igual que el de uso de descargas de barrera dieléctrica para la recuperación en situ de suelos contaminados (Aggelopoulos, y otros, 2013).

Una revisión de las aplicaciones de las descargas eléctricas en la disminución de compuestos orgánicos volátiles fue realizada por Vandenbroucke y otros (2011). En este trabajo se realiza un estudio comparativo de los resultados obtenidos por diferentes tipos de descargas, destacándose las descargas de barrera dieléctrica y las de corona. Para el caso específico del tratamiento de compuestos orgánicos volátiles de la industria de alimentos Preis, Klauson, y Gregor (2013), en una revisión más reciente concluyen que las descargas eléctricas son más eficientes en el tratamiento de las sustancias orgánicas volátiles, sin embargo consideran que la descargas de corona son mejores si se utilizan en combinación con tratamientos de catálisis y/o fotocatálisis. Un ejemplo específico de aplicación de la técnica descrita anteriormente en la que se usa una descarga de corona combinada con la interacción de la sustancia volátil con óxido de titanio sólido, se discute la posibilidad de uso en tubos de escape de motores diesel. (Mizuno, 2013). De igual manera mediante uso de una descarga de corona es posible eliminar el estireno proveniente de los aerosoles (Zhang, Feng, y otros, 2013), o la combinación de la descarga de barrera dieléctrica con técnicas sol-gel para hacer más eficiente la remoción del estireno (Zhang, Li, Sun, Jia, Lou, y Feng, 2014).

No menos interesantes resulta ser la aplicación de las descargas eléctricas en el tratamiento de desechos, una revisión de las técnicas más utilizadas es realizado por Gómez, Rani, y otros (2009), donde resulta interesante el uso de una antorcha plásmica que opera con una descarga de radiofrecuencia con acoplamiento inductivo. De igual forma una revisión extensa de los métodos usados para el tratamiento de aguas residuales basados en descargas eléctricas fue desarrollado por Jiang y otros (2013), la característica es la utilización de descargas de alto voltaje, logrando una recuperación rápida.

Finalmente existe un gran número de aplicaciones de las descargas eléctricas en el campo de la medicina (Laroussi, Kong, Morfill, y Stolz, 2012), algunas de ellas se describen en las revisiones sobre el plasma
Eco.Mat

Cúcuta-Colombia

Vol. 6

No. 1

Enero-Diciembre 2015 ISSN 1794-8231

ISSN 2462 - $8794 \mathrm{Web}$ PP: 6-21 
Eco.Mat

Cúcuta-Colombia

Vol. 6

No. 1

Enero-Diciembre 2015

ISSN 1794-8231

ISSN 2462 - $8794 \mathrm{We}$

PP: $6-21$
Descargas eléctricas y sus aplicaciones

físico en el tratamiento del cáncer realizado por Schegel, y otros (2013), o como la recopilación sobre las perspectivas de la aplicación del plasma físico en la endoscopia (Robert, y otros, 2013).

\section{Aplicaciones en Nanotecnología}

Lasdescargas eléctricas pueden sercontroladas al punto de conseguir las denominadas monocapas o multicapas atómicas en procesos de deposición, por lo tanto su utilidad en la nanotecnología es evidente (Lu, 2013, 94). Una revisión sobre las aplicaciones de las descargas en la síntesis de nano-estructuras fue realizado por Wang, Zhang, y Jiang (2013). Dentro de las múltiples aplicaciones esta la obtención de óxidos de titanio y de alúmina que son usados para darles propiedades catalíticas o de auto-limpieza bactericida a las superficies cerámicas.

\section{Referencias}

Abbas N.M., Solomon D.G., y Bahari Md. F., (2007), International of Machine Tools \& Manufacture, 47, 1214-1228.

Abbas N.M., Yusoff N., y Mahmod@Wahab R., (2012), Procedia Emgineering, 41, 16841688 .

Aggelopoulos C.A., Tsakiroglou C.D., Ognier S., y Cavadias S., (2013), Procedia Environmental Sciences, 18, 649-656.

Assarzadeh S., y Ghoreishi M., (2013), Procedia CIRP, 6, 463-468.

Bogaerts A., Neyts E., Gijbels R., y van der Mullen J., (2002), Spectrochimica Acta Part B, 57, 609-658.

Demellayer R., y Richard J., (2013), Procedia CIRP, 6, 89-94.

Devarani N., y Patowari P.K., (2013), Surface Modification of Graphite by Electrical Discharge Machine. LAP LAMBERT Academic Publishing, Deutschland, 67.

Dulce H.J., (2002), Implantación iónica tridimensional mediante descargas de alto voltaje a bajas presiones del dispositivo JUPITER, Tesis Doctoral, Universidad Industrial de Santander, 204.

Druyvesteyn M., Penning F., (1940), Rev. Mod. Phys., 12, 89-105.

Femsier R.F., Manheiner W.M., Meger R.A, Mathew J., et al, (1998), Phys. Plasmas, 5, 2140.

Gómez E., Rani, D.A., Cheeseman C.R., Deegan D., Wise M., y Boccaccini A.R., (2013), Journal of Hazardous Materials, 161, 614-628.

Grotjahn T., Aslambas Ö, Mee M., König M., y Meier S., (2013), Surface \& Coatings Technology, 273, 126-134.

Harry J.E., Introduction to Plasma Technology, Science, Engineering and Applications, WILEY-VCH, 2010, 215p.

Huddlestone R.H., Leonard S.L., (1965), Plasma diagnostic techniques, Academic Press, New York, 150.

Jiang B., Zheng J.,Qiu S., Wu M., Zhang Q., Yan Z., y Xue Q., (2014), Chemical Engineering Journal, 236, 348-368.

Joshi S.N., y Pande S.S., (2011), Applied Soft Computing, 11, 2743-2755.

Khanra A.K, y Pathak L.Ch., (2012), Electrical Discharge Machining (EDM) of Advanced Ceramics, LAP LAMBERT Academic Publishing, Deutschland, 38.

Klocke F., Schwade M., Klink A., Veselovac, y Kopp A., (2013), Procedia CIRP, 5, 88-93.

Krendel Y.E., (1977), Plasma sources of electrons, Atom, Moscow, 124.

Kumar S., Singh R., Singh T.P., y Sethi B.L., (2009), Journal of Materials Processing Technology, 209, 3675-3687. 
Laroussi M., Kong M.G., Morfill G., y Stolz W., (2012), Plasma Medicine, Applications of low-Temperature Gas Plasma in Medicine and Biology. Cambrige, New York, 346.

Levitsky S.M., Shanturin L.P. (1965), J. Techn. Phys., 35, 1182-1188.

Levitsky S.M., Shanturin L.P. (1967), J. Techn. Phys., 52, 350-356.

Lieberman M.A., Lichtenberg A.J., (1994), Principles of plasmas discharges and materials processing, John Wiley \& Sons Inc., New York, 165.

Lu, K., (2013), Nanoparticulate Materials, Synthesis, Characterization and Processing, WYLEY, New Jersey, 434.

Ludin D., y Pedersen H., (2013), Physics Procedia, 46, 3-11.

McClure G.W. (1961), Phys. Rev., 124, 969982.

Mizuno A., (2013), Catalysis Today, 211, 2-8.

Novikov A.A., (1983), Electron sources based on high voltage glow discharge, Energoatom, Moscow, 96.

Preis S., Klauson D., y Gregor A., (2013), Journal of Environmental Management, 114, 125-138.

Puncochar M., Rut B., y Chatterjee P.K., (2012), Procedia Engineering, 42, 420-430.

Robert E., Darny T., Dozias S., Collet G., Kieda C., y Pouvesle J.M., (2013), Clinical Plasma Medicine, 1, 8-16.

Roth R., Kuster F., y Wegener K., (2013), Procedia CIRP, 6, 338-343.

Schlegel J., Köritzer J., Boxhammer V., (2013), Clinical Plasma Medicine, 1, 2-7.

Schulze H.P., y Shätzing W., (2013), Procedia CIRP, 6, 58-63.
Singh A., (2012), Electrical Discharge Machining of Austempered Ductile Iron, LAP LAMBERT Academic Publishing, Deutschland, 145.

Singh H., y Shukla D.K., (2012), International Journal of Thermal Sciences, 59, 161-175.

Vandenbroucke A.M., Morent R., De Geyter N., y Leys C., (2011), Journal of Hazardous Materials, 195, 30-54.

Wang L., Zhang J., y Jiang W., (2013), Int. Journal of Refractory Metals and Hard Materials, 39, 103-112.

Weingärtner E., Kuster F., y Wegener K., (2012), Procedia CIRP, 2, 74-78.

Weir Ch., y Pantoja M., (2012), Electrostatic Discharge Sensitivity of Composite Energetic Materials, LAP LAMBERT Academic Publishing, Deutschland, 45.

Yang Y., Cho Y.I., y Fridman A., 2012, Plasma Discharge in Liquid: Water Treatment and Applications, CRC Press, New York, 189.

Zavialov M.A., Krendel Y.E., Navikov A.A., y Shanturin L.P., (1989), Plasma Processes in Electron Guns for Technological Applications, Energoatom, Moscow, 256p.

Zhang H., Li K., Sun T., Jia J., Lou Z., y Feng L., (2014), Chemical Engineering Journal, 241, 92-102.

Zhang X., Feng F., Li S., Tang X., Huang Y., Liu Z., y Yan K., (2013), Chemical Engineering Journal, 232, 527-533.

Zhang Y., Liu Y., Shen Y., Li Z., Ji R., y Wang F., (2013), Procedia CIRP, 6, 450-455.

Zhao Y., Kuneida M., y Abe K., (2013), Procedia CIRP, 6, 135-139. 